\title{
Cognitive reserve is associated with the functional organization of the brain in healthy aging: a MEG study
}

\author{
María E. López ${ }^{1,2+}$, Sara Aurtenetxe ${ }^{1,2+}$, Ernesto Pereda ${ }^{3}$, Pablo Cuesta ${ }^{1,2}$, Nazareth P. Castellanos ${ }^{1}$, \\ Ricardo Bruña ${ }^{1}$, Guiomar Niso' ${ }^{1}$ Fernando Maestú ${ }^{1,2}$ and Ricardo Bajo ${ }^{1,4}$ * \\ ${ }^{1}$ Laboratory of Cognitive and Computational Neuroscience (UCM-UPM), Centre for Biomedical Technology, Universidad Politécnica de Madrid, Madrid, Spain \\ 2 Department of Basic Psychology II, Complutense University of Madrid, Spain \\ ${ }^{3}$ Grupo de Ingeniería Eléctrica y Bioingeniería, Department of Industrial Engineering and Institute of Biomedical Technology, Universidad de La Laguna, La Laguna, \\ Tenerife \\ ${ }^{4}$ Departamento de Matemáticas, Universidad Internacional de La Rioja (UNIR), Logroño, Spain
}

Edited by:

Rodrigo Orlando Kuljiš, Zdrav Mozak

Limitada, Chile

Reviewed by:

Pablo Billeke, Universidad del

Desarrollo, Chile

Eduardo M. Castillo, Florida Hospital

for Children, USA

\section{*Correspondence:}

Ricardo Bajo, Laboratory of

Cognitive and Computational

Neuroscience (UCM-UPM), Centre

for Biomedical Technology,

Universidad Politécnica de Madrid,

Pozuelo de Alarcón, 28223 Madrid,

Spain

e-mail: ricardo.bajo@ctb.upm.es

these authors have contributed equally to this work.
The proportion of elderly people in the population has increased rapidly in the last century and consequently "healthy aging" is expected to become a critical area of research in neuroscience. Evidence reveals how healthy aging depends on three main behavioral factors: social lifestyle, cognitive activity, and physical activity. In this study, we focused on the role of cognitive activity, concentrating specifically on educational and occupational attainment factors, which were considered two of the main pillars of cognitive reserve (CR). Twenty-one subjects with similar rates of social lifestyle, physical and cognitive activity were selected from a sample of 55 healthy adults. These subjects were divided into two groups according to their level of $\mathrm{CR}$; one group comprised subjects with high CR ( 9 members) and the other one contained those with low CR (12 members). To evaluate the cortical brain connectivity network, all participants were recorded by Magnetoencephalography (MEG) while they performed a memory task (modified version of the Sternberg's Task). We then applied two algorithms [Phase Locking Value (PLV) and Phase Lag Index (PLI)] to study the dynamics of functional connectivity. In response to the same task, the subjects with lower CR presented higher functional connectivity than those with higher CR. These results may indicate that participants with low CR needed a greater "effort" than those with high CR to achieve the same level of cognitive performance. Therefore, we conclude that CR contributes to the modulation of the functional connectivity patterns of the aging brain.

Keywords: brain efficiency, cognitive reserve, functional connectivity, healthy aging, MEG

\section{INTRODUCTION}

Over the last decade, there has been increasing interest in the study of the cognitive decline associated with normal aging. In fact, demographic studies provide evidence of the progressive inversion of the population pyramid, such that it is expected that by the year $2100,22.3 \%$ of people will be aged 65 or over, as compared to a mere $7.6 \%$ in 2010 (source: United Nations). Thus, understanding aging is rightly considered a scientific and social priority warranting detailed investigation of the basic mechanisms associated with healthy aging.

It is well-known that cognitive capacity diminishes with aging (Fergus and Timothy, 1992; Grady, 2012). Evidence reveals that healthy aging is influenced by protective factors such as social lifestyle, cognitive and physical activity. In their review, Fratiglioni et al. (2004) claimed that "an active and socially integrated lifestyle in late life seems to protect against Alzheimer's disease and dementia."

Encompassed within cognitive activities, educational level and occupational attainment through life have been the two most studied factors and considered the strongest predictors of the maintenance of cognitive abilities in the aging process (see e.g.,
Stern et al., 1994; Anstey and Christensen, 2000; Ardila, 2000). These variables are involved in the concept of cognitive reserve (CR), which refers to the ability to tolerate age-related changes and disease-related pathologies in the brain, without developing clear clinical symptoms (Stern et al., 2012).

From a neurophysiological point of view, two non-mutually exclusive models have been proposed for the underlying reserve mechanism (Stern, 2002). At an anatomical level, the passive or brain reserve capacity (BRC) model considers direct measures of brain, such as brain volume or the number of synapses, to be factors underlying reserve, and assumes that larger brains present higher endurance capacity to neuropathology (Katzman et al., 1988; Mortimer et al., 2003). At a functional level, the active or CR's model considers the ability to recruit brain networks in an effective way to be the factor underlying reserve (Stern, 2002; Habeck et al., 2003; Scarmeas et al., 2003, 2004; Stern et al., 2005). According to this model, once pathological processes or cognitive decline begin to occur, CR uses the existing brain networks more efficiently to successfully perform a particular task (neural reserve) or alternative networks to maintain a normal cognitive status (neural compensation) (Stern et al., 2012). It is important 
to emphasize that although initially proposed as independent processes, brain reserve and CR concepts are not mutually exclusive and are both involved in providing protection against brain damage (Stern, 2006).

Several studies have tried to determine the anatomical and functional mechanisms/features underlying CR. These studies have provided evidence that CR contributes to modulate brain activity and effectiveness when executing cognitive tasks (Habeck et al., 2003; Scarmeas et al., 2003; Bartrés-Faz et al., 2009; SoléPadullés et al., 2009; Stern et al., 2012). Results from these studies reveal that, in order to achieve a similar level of performance during cognitive tasks, participants with low CR show higher brain activation than participants with high CR. However, to our knowledge, no research has studied the functional architecture of the networks involved in these processes.

Nowadays, one of the main approaches used for this purpose is the concept of functional connectivity. This term has been coined to describe how brain regions are coordinated to support higher cognitive functions (Friston, 1994, 2001; Sporns et al., 2004). Functional connectivity reflects the statistical dependencies between two physiological signals, which provide relevant information about functional interactions between the corresponding brain regions. Long-range synchronization between the oscillatory activity of brain signals originating in relatively distant neuronal populations has been proposed as the mechanism for communication and integration of information in the brain (Varela et al., 2001; Fries, 2005), and it is also present during spontaneous oscillatory activity (Hipp et al., 2012). Therefore, the characterization of functional connectivity is a suitable approach to study brain functioning (Singer, 1999; Buzsáki and Draguhn, 2004), and hence the CR.

Magnetoencephalography (MEG) facilitates such an approach, with its signals providing a direct measure of neuronal activity, which allows the dynamics of the cortical networks underlying cognition to be studied. By applying functional connectivity indexes to MEG data, it is possible to infer the topology and efficiency of functional neural networks underlying cognitive processes (De Haan et al., 2012; Siegel et al., 2012). However, as far as we know, no research has used functional connectivity to analyze MEG signals underlying CR during the execution of a cognitive task.

Thus, this work aimed to study the relationship between CR, brain dynamics and cognitive performance in healthy aging. As educational and occupational attainment throughout life have been proposed as adequate proxies for brain and CR models (Stern, 2002, 2009), we chose these two variables to characterize participants in this study. Therefore, participants were first divided into two groups according to their score on a CR index (CRI) proposed by Garibotto et al. (2012), which was based on both proxy measures of reserve (see Materials and Methods for the explanation). To ensure adequate isolation of these two variables, we controlled the other factors which may be also involved in $\mathrm{CR}$, such as social, physical, and cognitive activities realized at present. Then, MEG signals were recorded during the execution of a cognitive task. Specifically, we chose a memory task (Sternberg's modified Task) because cognitive capacity diminishes with aging, with memory decline being one of its main features (Grady, 2012). We then used two phase synchronization
(PS) indexes [Phase Locking Value (PLV) and Phase Lag Index (PLI)], which assess the degree of functional connectivity between oscillatory narrow-band signals, in order to characterize the functional topology of the neural network of each group while performing the memory task.

Based on the existing literature, we hypothesized that participants with lower educational and occupational attainment would reach a similar level of performance but showing higher connectivity values in most of the frequency bands analyzed, as compared to participants with higher educational and occupational attainment.

\section{MATERIALS AND METHODS PARTICIPANTS}

Twenty-one healthy adults enrolled in the study. All of them were recruited from the "Geriatric Unit of the University Hospital San Carlos" (Madrid, Spain). All subjects fulfilled the following inclusion criteria: (1) right handed (Oldfield, 1971); (2) native Spanish speakers; (3) between 65 and 85 years old; (4) a Mini-Mental State Examination (MMSE, Lobo et al., 1979) score greater than 28; (5) no memory impairment documented by delayed recall from the Logical Memory II subtest of the Wechsler Memory Scale Revised (WMS-III-R; Wechsler, 1987), (cut-off scores $>16$ for participants with more than 15 years of education; $>8$ for participants with 8-15 years of education); and (6) normal daily living activities (assessed with the Spanish version of the Functional Assessment Scale Pfeffer et al., 1982). Exclusion criteria included: (1) medical history of psychiatric or any other neurological disease; (2) psychoactive drugs consumption; and (3) severe sensory or comprehension deficits.

We considered the educational level and the occupational attainment to classify the subjects according to a scale (see Figure 1). Education was measured depending on the formal education attained and occupational attainment taking into account the main professional activity carried out during the active life of each participant. Moreover, current physical, cognitive, and social activities performed by each participant were subsequently assessed by a self-reported questionnaire, where the score was based on the weekly frequency of each activity (see Table 1). In the physical category, we included the following activities: swimming, running, walking, or going to the gym; in the cognitive category these others: knitting, listening to music or to the radio, reading (books, magazines, newspapers), playing games (cards, parcheesi), going to classes, to a club or center, watching TV, going to the cinema, theater, or sport events, participating in cultural activities or other hobbies. Finally, in the social category we considered: visiting friends or family and participating in social activities.

In order to study the effect of education and occupational attainment (CRI factors) during the execution of a cognitive task, the initial 55 participants were matched for physical, cognitive, and social activities during late-life. Therefore, 21 participants were finally included in the study. Then, they were divided in two different subgroups according to the CRI, which was calculated by adding two independent categories: the educational level (maximum score of 5) and the occupational attainment (maximum score of 5) (Garibotto et al., 2012), Table 2 summarizes the features of each group. Based on this CRI, we considered that 


\section{COGNITIVE RESERVE INDEX \\ Educational level + Occupation}

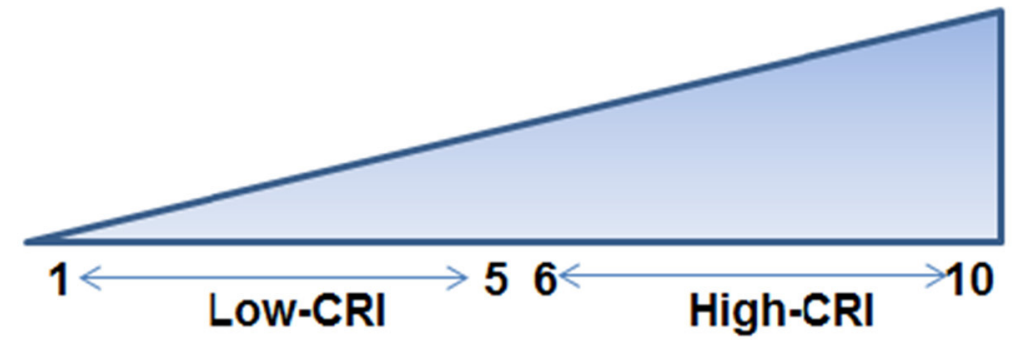

EDUCATIONAL LEVEL

OCCUPATIONAL ATTAINMENT

$1=$ Illiterate/ Functional illiterate $\quad 1=$ Housewife

2=PrimaryStudies 2=Non-Qualified

3=Elemental Studies 3= Qualified

4= High SchoolStudies $\quad 4=$ Technical/Professional

$\mathbf{5}=$ Superior studies $\quad 5=$ Business Management $/$ Research

FIGURE 1 | Cognitive reserve index (CRI) calculation. Educational level was grouped into five levels: (1) illiterate/functional illiterate, (2) primary studies, (3) elemental studies, (4) high school studies, (5) superior studies.
Occupational attainment was divided into five levels: (1) housewife, (2) non-qualified, (3) qualified; (4) technical/professional; (5) business management/research.

Table 1 | Description of the scores of cognitive, physical, and social activities per week.

\section{Activities}

Cognitive

Physical

Social

$\begin{array}{ll}0 \text { (0 points }) & 1-3 \text { (1 point }) \\ 0 \text { (0 points }) & 1-2 \text { (1 point }) \\ 0 \text { (0 points }) & 1-2 \text { (1 point })\end{array}$

4-7 (2 points)

3-5 (2 points)

8-10 (3 points)

$6-7$ (3 points)

$>5$ (3 points)
Hours per week

a subject with scores between 1 and 5 belonged to the low CRI group, whereas those with scores between 6 and 10 were classified into the high CRI group (see Figure 1). With this procedure, the low CRI group was finally formed by 12 subjects, whereas the high CRI group was formed by 9 subjects (see Table 2).

Before MEG recordings, all participants gave written informed consent to participate in the study, which was approved by the Local Ethics Committee.

\section{STIMULI AND TASK}

A modified version of the Sternberg's letter probe task (deToledo-Morrell et al., 1991) was used (see Figure 2). A set of five letters was presented to the participants, who were then asked to keep them in mind. After the presentation of the five letter set, a series of single letters $(500 \mathrm{~ms}$ in duration, with a random ISI between 2 and $3 \mathrm{~s}$ ) was presented one at a time, and the participants were asked to press a button with their right hand when a member of the initial set was detected. The list consisted of 250 letters, in which half were targets (i.e., they were present in the initial set), and half distracters (i.e., they were not). All participants completed a training session before the actual test, which did not start until each subject demonstrated that he/she could remember the five letter set. Letters were projected through a LCD video projector (SONY VPLX600E), situated outside of the shielded 
Table 2 | Description of the mean and standard deviations of each group.

\begin{tabular}{lrcc}
\hline & High-CRI & Low-CRI & Sig. \\
\hline Age & $67.3 \pm 7.4$ & $69.7 \pm 6.6$ & $p>0.05$ \\
Educ. level & $4.5 \pm 0.7$ & $2.4 \pm 0.7$ & $p<0.05^{*}$ \\
Occupation & $4.5 \pm 0.5$ & $1.6 \pm 0.5$ & $p<0.05^{*}$ \\
CRI & $9 \pm 0.6$ & $3.7 \pm 1.1$ & $p<0.05^{*}$ \\
Cognitive act. & $4.2 \pm 2.3$ & $2.8 \pm 1.4$ & $p>0.05$ \\
Physical act. & $2.4 \pm 1.5$ & $2.7 \pm 1$ & $p>0.05$ \\
Social act. & $1.6 \pm 1.2$ & $1.7 \pm 1$ & $p>0.05$ \\
Acc. Smaqe & $114 \pm 10.7$ & $106 \pm 14.1$ & $p>0.05$
\end{tabular}

Statistically significant values are $p<0.05^{*}$. Groups (High and Low CRI) differ in educational level and occupation and therefore, in $C R$, but they are not different otherwise. Accuracy of the memory task performance (Acc. Smaqe) did not differ significantly between groups.

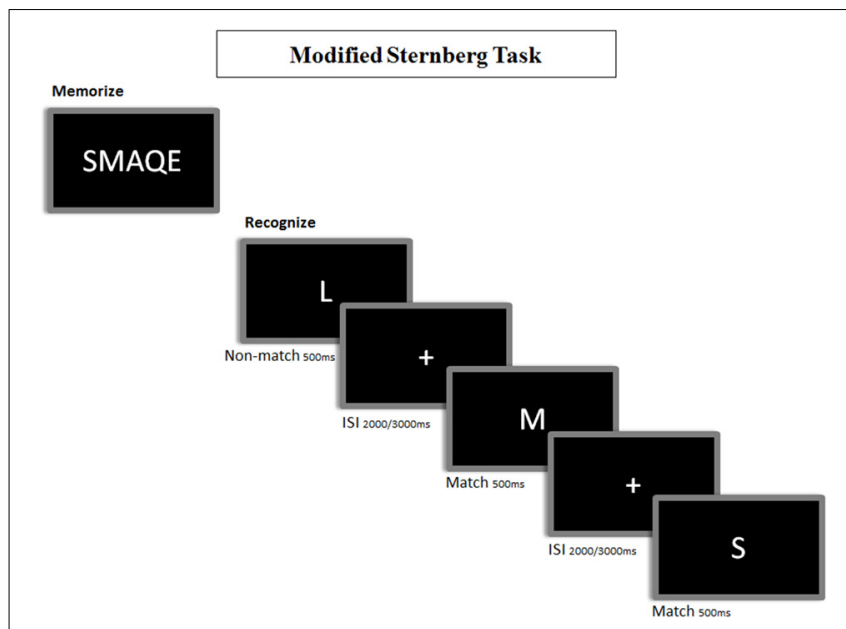

FIGURE 2 | Representation of the memory task paradigm. In the encoding phase, participants are instructed to memorize 5 letters (i.e.: "SMAQE"). In the recognition phase, participants are instructed to make a match/non-match button-press to indicate that the presented letter matched any of the encoded ones.

room onto a series of in-room mirrors, the last of which was suspended approximately 1 meter above the participant's face. The letters subtended 1.8 and 3 degrees of horizontal and vertical visual angle, respectively.

\section{MEG DATA COLLECTION}

MEG signals were recorded during the execution of the modified Sternberg's task described above, with a $254 \mathrm{~Hz}$ sampling frequency and a band pass of 0.5 to $50 \mathrm{~Hz}$, using a 148 channel whole-head magnetometer (MAGNES $2500 \mathrm{WH}, 4 \mathrm{D}$ Neuroimaging) confined in a magnetically shielded room. An environmental noise reduction algorithm using reference channels at a distance from the MEG sensors was applied to the data. Thereafter, single trial epochs were visually inspected by an experienced investigator, and epochs containing visible blinks, eye movements, or muscular artifacts were excluded from further analysis. Artifact free epochs from each channel were then classified into four different categories, according to the subject's performance in the experiment: hits, false alarms, correct rejections, and omissions. Only hits were considered for further analysis, because we were interested in evaluating the functional connectivity patterns that support recognition success. Thirtyfive epochs per subject were used to calculate PLV and PLI values. This lower bound was determined by the participant with least epochs. So, to have an equal number of epochs across participants, the 35 epochs were randomly chosen from each of the other participants.

\section{MEG DATA ANALYSIS PLV and PLI}

All epochs were band-pass filtered off-line in frequency bands of $4 \mathrm{~Hz}$ in the range between 4 and $44 \mathrm{~Hz}: 4-8 \mathrm{~Hz}, 8-12 \mathrm{~Hz}$, $12-16 \mathrm{~Hz}, \ldots, 40-44 \mathrm{~Hz}$. Subsequently, PLV and PLI values were calculated for each of the 351 -s epochs to estimate the degree of PS within each of these frequency bands for each of the $148^{*} 147 / 2$ sensors pairs using the HERMES toolbox (Niso et al., 2013). PLV makes use of the relative phase difference (Lachaux et al., 1999; Mormann et al., 2000). It is defined as:

$$
\begin{aligned}
P L V= & \left|\left\langle e^{i \Delta \phi\left(t_{n}\right)}\right\rangle\right|=\left|\frac{1}{N} \sum_{n=1}^{N} e^{i \Delta \phi\left(t_{n}\right)}\right| \\
& =\sqrt{\langle\cos \Delta \phi(t)\rangle^{2}+\langle\sin \Delta \phi(t)\rangle^{2}}
\end{aligned}
$$

where $<.>$ indicates time average and $\Delta \phi(t)$ is the cyclic relative phase at time $t$, i.e., the difference between the phase of signals $\mathrm{x}(\mathrm{t})$ and $\mathrm{y}(\mathrm{t})$ wrapped to the interval $[0,2 \pi)$

The PLV estimates how the relative phase is distributed over the unit circle. When there is strong PS between $\mathrm{x}(\mathrm{t})$ and $\mathrm{y}(\mathrm{t})$, $\Delta \phi(t)$ occupies a small portion of the circle and the PLV is close to 1 , whereas if the signals are not synchronized, the relative phase would spread out all over the unit circle and the PLV would remain low. Note that PLV is not robust against the presence of common sources (e.g., volume conduction effects).

Instead, the PLI (Stam et al., 2007), discards phase differences that centered around $0 \bmod \pi$ in order to be robust against the presence of common sources (volume conduction).

$$
P L V=\left|\left\langle\operatorname{sign}\left(\Delta \phi\left(t_{n}\right)\right)\right\rangle\right|=\left|\frac{1}{N} \sum_{n=1}^{N} \operatorname{sign}\left(\Delta \phi\left(t_{n}\right)\right)\right|
$$

The range of this index is $0 \leq P L I \leq 1$. (0): no coupling or coupling with a phase difference centered around 0 or $\pi,(1)$ : perfect phase locking at a value of $\Delta \phi(t)$ different from $0 \bmod \pi$.

\section{STATISTICAL ANALYSIS}

Once PLV and PLI values were calculated, a between-group Mann-Whitney $U$-test (non-parametric test) was performed for each pair of sensors and the two groups of subjects [high CR (9 members) and low CR (12 members)]. Thus, we obtained a symmetric squared matrix $(148 \times 148)$ containing all the corresponding $\mathrm{p}$ values.

Subsequently, to avoid the multiple comparisons problem, we performed a non-parametric permutation testing (Ernst, 2004). 
This was done by randomly dividing the participants into two sets, matching the numbers of the original groups (9 and 12). Besides, for each subject, PLV and PLI values were randomized.

Then, a Mann-Whitney $U$-test between each pair of sensors was then carried out between these two newly created groups. The procedure was repeated 2000 times and the $p$-value from each test for sensor pair was calculated to obtain a $p$-value distribution for each channel pair. We then identified the 1th percentile of each distribution, and only those $p$-values below that threshold were considered significant.

\section{RESULTS}

\section{DEMOGRAPHIC RESULTS}

High- and low-CRI participants were matched in age $\left[t_{(20)}=0.8, p=0.4\right]$, cognitive $\left[t_{(20)}=1.6, p=0.1\right]$, physical $\left[t_{(20)}=0.6, p=0.5\right]$, and social $\left[t_{(20)}=0.1, p=0.9\right]$ activities. Analysis of the task performance revealed no differences between groups $\left[t_{(20)}=1.4, p=0.1\right]$.

\section{CONNECTIVITY RESULTS}

Statistical analysis of both PLV and PLI values revealed an overall increase in connectivity in the low CRI participants, compared to the high CRI subjects in the following frequency bands: 4-8 Hz (theta), $8-12 \mathrm{~Hz}$ (alpha), $12-16 \mathrm{~Hz}$ (beta 1 ), and $16-20 \mathrm{~Hz}$ (beta 2) (see Figure 3 and Table 3), but located in different sensors (see Figure 4). Thus, differences in synchronization in theta band between both groups were observed between right fronto-occipital sensors and between right parietal-occipital sensors. In alpha band low CRI participants showed higher synchronization values between fronto-temporal sensors of the left hemisphere and within occipital sensors. Differences in beta 1 band were observed between fronto-left parietal sensors and right temporo-occipital sensors and within left temporal sensors. Finally, differences in beta 2 band were observed between frontoleft temporal sensors, between left temporo-parietal sensors,

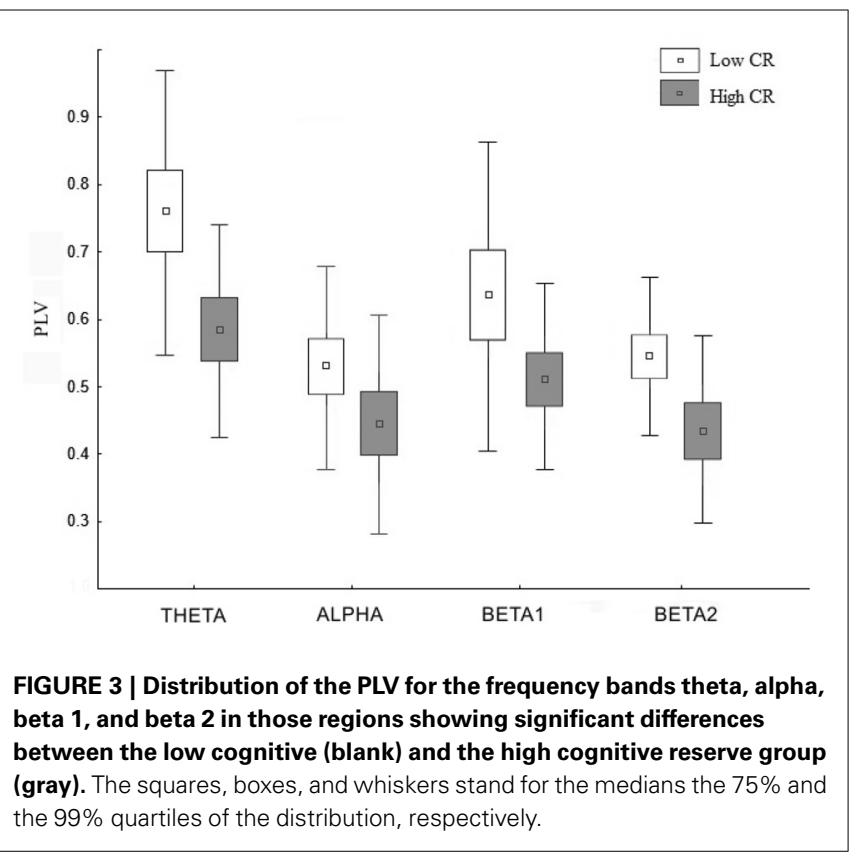

between right temporo-occipital areas y and within left temporal sensors (Figure 4).

\section{DISCUSSION}

This study explored the relation between brain activity architecture and educational and occupational attainment throughout life (as assessed by an index of CR, the CRI), during the performance of a memory task in healthy aging.

For this purpose, healthy elderly subjects were grouped according to their different CR profiles, which were based on the two principal factors of CR. Thus, we studied two different groups based on the CRI: low and high CR, having controlled for age, physical, cognitive, and social current activities; in order to specifically assess the influence of CR on the organization of the functional networks. Then, we applied functional connectivity analysis to MEG time series by using two PS indexes, which are a robust measure of the extent of information integration in the brain (Stam and van Straaten, 2012). Since it is generally accepted that the process of normal aging impairs synaptic function and neuronal communication (Morrison and Baxter, 2012), we proposed that these methods would provide new insights about how functional neural networks are shaped by CR in elderly.

Increased functional connectivity may be related to network efficiency (Buldú et al., 2011). In an elegant study Palva et al. (2010) proved that during performance of a working memory task, synchronization increased with memory load in young subjects. That is, the more difficult the task, the more tightly connected the brain network has to be to perform successfully. Similarly, (Zarahn et al., 2007) compared brain activity in young and elderly subjects during a Sternberg task that progressively increased in terms of memory load. They found that elderly subjects needed higher activation of their neuronal networks than the young subjects, due to lower efficiency of the elderly subjects' neuronal networks. These findings appeared to substantiate the fact that, to successfully perform the same task, subjects with lower educational and occupational attainment present increased brain connectivity as compared to subjects with higher level in these two variables. These results lead us to claim that, since individuals with a lower CRI need increased connectivity strength to perform the same cognitive task, their cortical networks are less efficient. That is, they need to overuse the brain functional networks to achieve a similar performance on the memory task, which would imply a decrease in network efficiency.

In summary, efficiency refers to the change in neural activity occurring with a change in task demand. Thus, greater efficiency would be associated with the recruitment of less neural activity and therefore less energy loss. As a consequence, under similar task performance, the more efficient network would be the one presenting lower brain synchronization and energy loss (for similar interpretations see Stern et al., 2005, 2012; Steffener and Stern, 2012). The discussion on connectivity related to efficiency is a relatively novel issue and has not yet been clarified. Bajo et al. (2010) showed that inter-hemispheric synchronization values increase reflected a compensatory mechanism for the lack of memory network efficiency in patients suffering from mild cognitive impairment (MCI). A network analysis revealed a nonefficient organization (Buldú et al., 2011) indicating that the 
Table 3 | Percentage over total number of links (pairs of sensors) (Nlinks $=148 * 147 / 2=10870$ ), showing significant differences between both groups for the frequency bands studied (theta, alpha, beta 1, and beta 2).

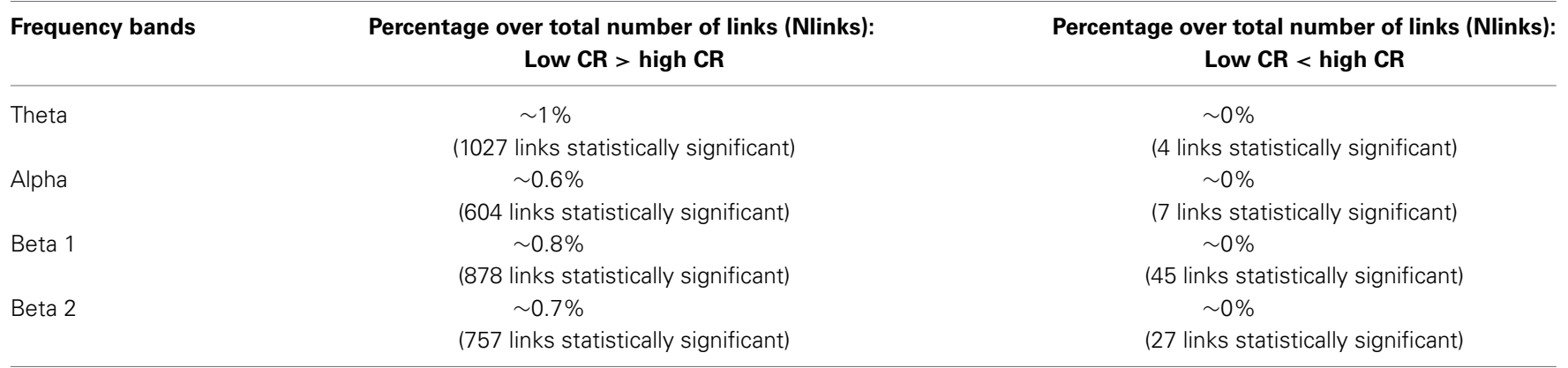

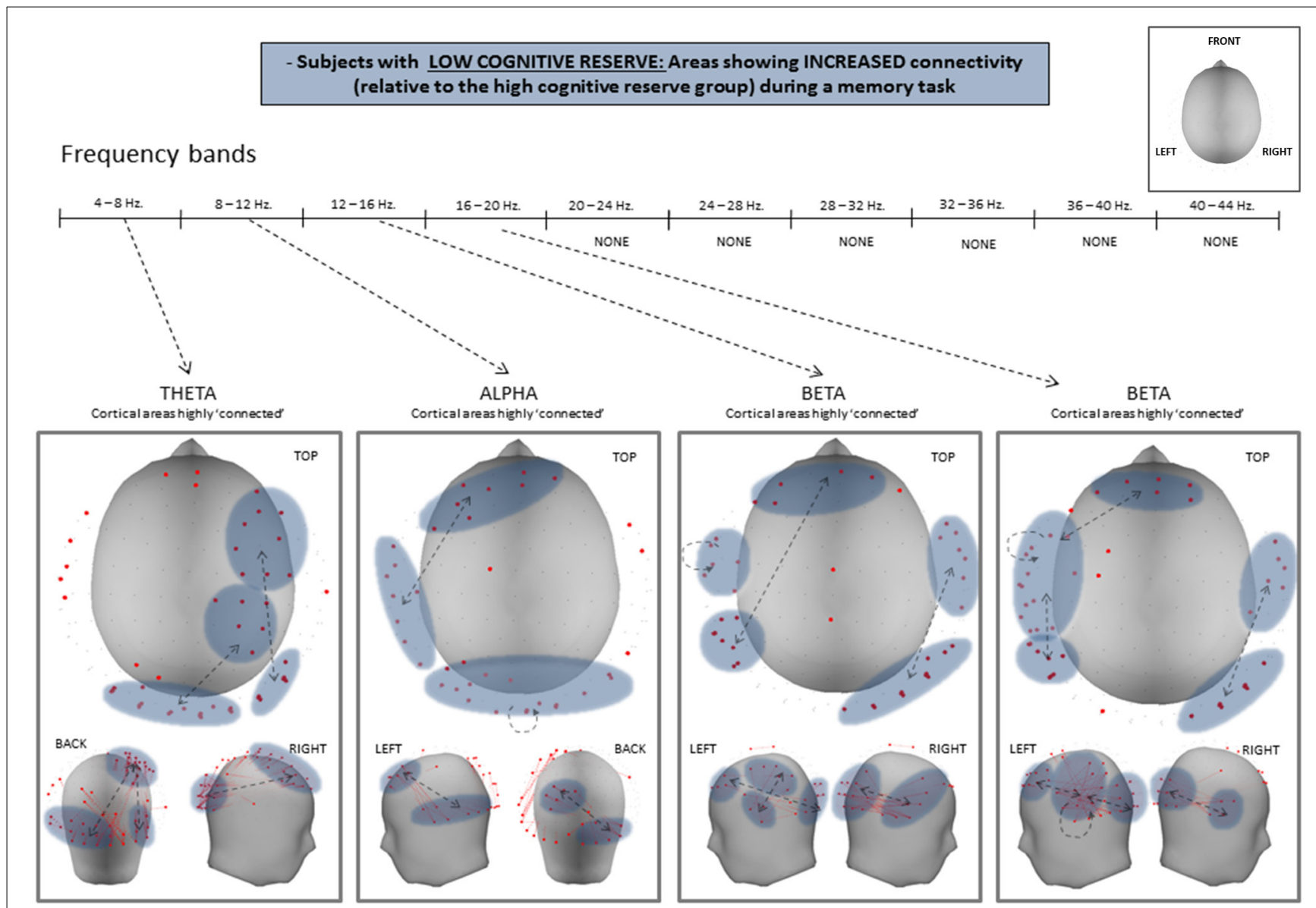

FIGURE 4 | Statistical differences of PLV between high- and low-CRI subjects in theta, alpha, beta 1, and beta 2 frequency bands.

increased functional connectivity it is not always associated with a better network functioning.

Modulation of brain activity during cognitive performance has also been found when decomposing EEG/MEG signals into spectral frequency bands. An increase in theta band activity has been observed during memory performance (Klimesch, 1996; Kim et al., 2012), especially in medial prefrontal regions (Raghavachari et al., 2001; Langer et al., 2013). Furthermore, coupling of theta band activity between several brain regions has been correlated with improving task execution (Axmacher et al., 2010; Fell and Axmacher, 2011; Langer et al., 2013). In a similar manner, alpha band activity has been related to active cognitive processes during memory tasks (Palva and Palva, 2007) especially between prefrontal and parietal regions (Grimault et al., 2009). Furthermore, an increase in alpha band activity during the detection of previously presented items suggests that this band plays a role 
in recognition processes (Van Strien et al., 2005). The activity in theta and alpha band has been associated with the amount of information to be retained (Klimesch, 1999; Jensen and Tesche, 2002; Jensen et al., 2002; Palva et al., 2010; Meeuwissen et al., 2011; Palva and Palva, 2011). Recently, Geerligs et al. (2014) compared young and elderly people and showed that an increase in connectivity in both alpha and beta bands was related to a better performance in older participants only, suggesting that this is an active compensation mechanism employed to maintain adequate performance. It seems likely that this normal increase in connectivity that is used as a compensatory mechanism in the elderly is being overused by low CRI subjects. Thus, these three frequency bands are related to the memory process and seem to be overactive in the CRI group, indicating a non-efficient organization of the functional networks.

Our findings agree with the aforementioned literature, inasmuch as they revealed that subjects with lower CRI present greater connectivity than those with higher CRI in the alpha and beta bands in the left hemisphere and in the theta band of the right hemisphere. This increased connectivity was observed in both anterior (prefrontal) and posterior (temporal, parietal, and occipital) regions. The present results regarding a high connectivity in low CRI participants over the left hemisphere accounts for the verbal nature of the memory task utilized. Given that all of our subjects were right-handed, the recruitment of the dominant hemisphere for the execution of the task reflects its role in mediating verbal memory processes. In the case of the right hemisphere, the recruitment of brain regions here seems to indicate compensatory mechanisms that contribute to the performance of the task. Brain bilateral activity in response to verbal information has been found in studies comparing young and elderly subjects and interpreted as a compensatory mechanism in the context of the HAROLD model (Cabeza et al., 2002; Reuter-Lorenz, 2002). Moreover, the over-recruitment of brain regions in elderly subjects has been observed when achieving the same accuracy as younger subjects (Morcom, 2003). Considering this context, and in agreement with our initial hypothesis, elderly individuals with lower CRI need to over-recruit more cortical networks, showing a lesser efficient brain functioning, to achieve the same level of cognitive performance as elderly individuals with higher CRI.

From a methodological point of view, the fact that our results are consistent with the two PS indexes used (similar results were obtained for both indexes, then only PLV outcomes were showed, Figure 4), which measure the existence of different types of coherent phase relationship between two signals, further supports the validity of these outcomes. In fact, the PLV does not distinguish between zero (i.e., 0 or $\pi$ relative phase) and non-zero lag interdependence between two signals, the former of these phase relationships being normally ascribed to a mixture of one or more common neural source in the two sensors analyzed (Nolte et al., 2004). In contrast, PLI is nonzero if and only if this phase relationship is asymmetric, i.e., there is a certain time lag between the data, which is regarded as a sign of true direct connectivity (Nolte et al., 2004; Stam et al., 2007). However, zero-lag synchronization between two distant systems might occur if they are indirectly connected through a common relay (Fischer et al., 2006). This type of synchronization would result in a zero PLI between the two connected systems, which would not be the result of a common source (i.e., the same neural activity of a single brain area) measured on both of them. Yet such an indirect connection between cortical areas (which could be only detected using the PLV) with some deep brain structure acting as a dynamic relay, cannot certainly be ruled out (Gollo et al., 2011). This connectivity would indeed have a neurological origin rather than being a volume conduction artifact. Thus, the combined use of both indexes provides complementary, rather than confounding, information and our results suggest that both mechanisms (direct connectivity as assessed by the PLI and indirect connectivity / increased common source activity as assessed by the PLV) should be taken into account for measuring the brain efficiency during the execution of a cognitive task in subjects with different CR level.

One main limitation when interpreting the present results considers the size of the sample. For this reason, future studies should include a larger number of participants. Additionally, exploration of how cognitive demands shape the functional networks of the brain will contribute to characterize how functional neural networks are influenced by CR.

The current data shows the importance of quantifying the relation between the CR and the functional connectivity structure of the aging brain, and to evaluate its role in preventive and therapeutic strategies to achieve the goal of healthy aging.

\section{ACKNOWLEDGMENTS}

This study was supported by: a predoctoral fellowship from the Basque Government to Sara Aurtenetxe; a predoctoral fellowship from the Ministry of Education of Spain (FPU AP-2008-00175) and by the "PSI2009-14415-C03 project of the Spanish Ministry of Science and Innovation" to María E. López and Pablo Cuesta. The authors also acknowledge the financial support of the grants: TEC2012-38453-C04-01 and -03 of the MINECO, and PSI201238375-C03-01.

\section{REFERENCES}

Anstey, K., and Christensen, H. (2000). Education, activity, health, blood pressure and apolipoprotein $\mathrm{E}$ as predictors of cognitive change in old age: a review. Gerontology 46, 163-177. doi: 10.1159/000022153

Ardila, A. (2000). Age-related cognitive decline during normal aging: the complex effect of education. Arch. Clin. Neuropsychol. 15, 495-513. doi: 10.1016/S08876177(99)00040-2

Axmacher, N., Henseler, M. M., Jensen, O., Weinreich, I., Elger, C. E., and Fell, J. (2010). Cross-frequency coupling supports multi-item working memory in the human hippocampus. Proc. Natl. Acad. Sci. U.S.A. 107, 3228-3233. doi: 10.1073/pnas.0911531107

Bajo, R., Maestú, F., Nevado, A., Sancho, M., Gutiérrez, R., Campo, P., et al. (2010). Functional connectivity in mild cognitive impairment during a memory task: implications for the disconnection hypothesis. J. Alzheimers Dis. 22, 183-193. doi: 10.3233/JAD-2010-100177

Bartrés-Faz, D., Solé-Padullés, C., Junqué, C., Rami, L., Bosch, B., Bargalló, N., et al. (2009). Interactions of cognitive reserve with regional brain anatomy and brain function during a working memory task in healthy elders. Biol. Psychol. 80, 256-259. doi: 10.1016/j.biopsycho.2008.10.005

Buldú, J. M., Bajo, R., Maestú, F., Castellanos, N., Leyva, I., Gil, P., et al. (2011). Reorganization of functional networks in mild cognitive impairment. PLoS ONE 6:e19584. doi: 10.1371/journal.pone.0019584

Buzsáki, G., and Draguhn, A. (2004). Neuronal oscillations in cortical networks. Science 304, 1926-1929. doi: 10.1126/science.1099745 
Cabeza, R., Anderson, N. D., Locantore, J. K., and McIntosh, A. R. (2002). Aging gracefully compensatory brain activity. Neuroimage 17, 1394-1402. doi: 10.1006/nimg.2002.1280

De Haan, W., van der Flier, W. M., Koene, T., Smits, L. L., Scheltens, P., and Stam, C. J. (2012). Disrupted modular brain dynamics reflect cognitive dysfunction in Alzheimer's disease. Neuroimage 59, 3085-3093. doi: 10.1016/j.neuroimage.2011.11.055

deToledo-Morrell, L., Evers, S., Hoeppner, T. J., Morrell, F., Garron, D. C., and Fox, J. H. (1991). A "stress" test for memory dysfunction. Electrophysiologic manifestations of early Alzheimer's disease. Arch. Neurol. 48, 605-609. doi: 10.1001/archneur.1991.00530180061018

Ernst, M. D. (2004). Permutation methods: a basis for exact inference. Stat. Sci. 19, 676-685. doi: 10.1214/088342304000000396

Fell, J., and Axmacher, N. (2011). The role of phase synchronization in memory processes. Nat. Rev. Neurosci. 12, 105-118. doi: 10.1038/nrn2979

Fergus, I. M. C., and Timothy, A. S. (1992). The Handbook of Aging and Cognition. Hillsdale, NJ; England: Lawrence Erlbaum Associates, Inc.

Fischer, I., Vicente, R., Buldú, J. M., Peil, M., Mirasso, C. R., Torrent, M. C., et al. (2006). Zero-lag long-range synchronization via dynamical relaying. Phys. Rev. Lett. 97:123902. doi: 10.1103/PhysRevLett.97.123902

Fratiglioni, L., Paillard-Borg, S., and Winblad, B. (2004). An active and socially integrated lifestyle in late life might protect against dementia. Lancet Neurol. 3, 343-353. doi: 10.1016/S1474-4422(04)00767-7

Fries, P. (2005). A mechanism for cognitive dynamics: neuronal communication through neuronal coherence. Trends Cogn. Sci. 9, 474-480. doi: 10.1016/j.tics.2005.08.011

Friston, K. J. (1994). Functional and effective connectivity in neuroimaging: a synthesis. Hum. Brain Mapp. 2, 56-78. doi: 10.1002/hbm.460020107

Friston, K. J. (2001). Brain function, nonlinear coupling, and neuronal transients. Neuroscientist 7, 406-418. doi: 10.1177/107385840100700510

Garibotto, V., Borroni, B., Sorbi, S., Cappa, S. F., Padovani, A., and Perani, D. (2012). Education and occupation provide reserve in both ApoE $\varepsilon 4$ carrier and noncarrier patients with probable Alzheimer's disease. Neurol. Sci. 33, 1037-1042. doi: 10.1007/s10072-011-0889-5

Geerligs, L., Maurits, N. M., Renken, R. J., and Lorist, M. M. (2014). Reduced specificity of functional connectivity in the aging brain during task performance. Hum. Brain Mapp. 35, 319-330. doi: 10.1002/hbm.22175

Gollo, L. L., Mirasso, C. R., Atienza, M., Crespo-Garcia, M., and Cantero, J. L. (2011). Theta band zero-lag long-range cortical synchronization via hippocampal dynamical relaying. PLoS ONE 6:e17756. doi: 10.1371/journal.pone. 0017756

Grady, C. (2012). The cognitive neuroscience of ageing. Nat. Rev. Neurosci. 13, 491-505. doi: 10.1038/nrn3256

Grimault, S., Robitaille, N., Grova, C., Lina, J.-M., Dubarry, A.-S., and Jolicoeur, P. (2009). Oscillatory activity in parietal and dorsolateral prefrontal cortex during retention in visual short-term memory: additive effects of spatial attention and memory load. Hum. Brain Mapp. 30, 3378-3392. doi: 10.1002/hbm.20759

Habeck, C., Hilton, H. J., Zarahn, E., Flynn, J., Moeller, J., and Stern, Y. (2003). Relation of cognitive reserve and task performance to expression of regional covariance networks in an event-related fMRI study of nonverbal memory. Neuroimage 20, 1723-1733. doi: 10.1016/j.neuroimage.2003.07.032

Hipp, J. F., Hawellek, D. J., Corbetta, M., Siegel, M., and Engel, A. K. (2012). Large-scale cortical correlation structure of spontaneous oscillatory activity. Nat. Neurosci. 15, 884-890. doi: 10.1038/nn.3101

Jensen, O., Gelfand, J., Kounios, J., and Lisman, J. E. (2002). Oscillations in the alpha band $(9-12 \mathrm{~Hz})$ increase with memory load during retention in a short-term memory task. Cereb. Cortex 12, 877-882. doi: 10.1093/cercor/ 12.8.877

Jensen, O., and Tesche, C. D. (2002). Frontal theta activity in humans increases with memory load in a working memory task. Eur. J. Neurosci. 15, 1395-1399. doi: 10.1046/j.1460-9568.2002.01975.x

Katzman, R., Terry, R., DeTeresa, R., Brown, T., Davies, P., Fuld, P., et al. (1988). Clinical, pathological, and neurochemical changes in dementia: a subgroup with preserved mental status and numerous neocortical plaques. Ann. Neurol. 23, 138-144. doi: 10.1002/ana.410230206

Kim, S.-P., Kang, J.-H., Choe, S.-H., Jeong, J. W., Kim, H. T., Yun, K., et al. (2012). Modulation of theta phase synchronization in the human electroencephalogram during a recognition memory task. Neuroreport 23, 637-641. doi: 10.1097/WNR.0b013e328354afed
Klimesch, W. (1996). Memory processes, brain oscillations and EEG synchronization. Int. J. Psychophysiol. 24, 61-100. doi: 10.1016/S0167-8760(96)00057-8

Klimesch, W. (1999). EEG alpha and theta oscillations reflect cognitive and memory performance: a review and analysis. Brain Res. Brain Res. Rev. 29, 169-195. doi: 10.1016/S0165-0173(98)00056-3

Lachaux, J. P., Rodriguez, E., Martinerie, J., and Varela, F. J. (1999). Measuring phase synchrony in brain signals. Hum. Brain Mapp. 8, 194-208.

Langer, N., von Bastian, C. C., Wirz, H., Oberauer, K., and Jäncke, L. (2013). The effects of working memory training on functional brain network efficiency. Cortex 49, 2424-2438. doi: 10.1016/j.cortex.2013.01.008

Lobo, A., Ezquerra, J., Gómez Burgada, F., Sala, J. M., and Seva Díaz, A. (1979). [Cognocitive mini-test (a simple practical test to detect intellectual changes in medical patients)]. Actas Luso Esp. Neurol. Psiquiatr. Cienc. Afines 7, 189-202.

Meeuwissen, E. B., Takashima, A., Fernández, G., and Jensen, O. (2011). Increase in posterior alpha activity during rehearsal predicts successful long-term memory formation of word sequences. Hum. Brain Mapp. 32, 2045-2053. doi: $10.1002 / \mathrm{hbm} .21167$

Morcom, A. M. (2003). Age effects on the neural correlates of successful memory encoding. Brain 126, 213-229. doi: 10.1093/brain/awg020

Mormann, F., Lehnertz, K., David, P., and Elger, C. E. (2000). Mean phase coherence as a measure for phase synchronization and its application to the EEG of epilepsy patients. Phys. D Nonlinear Phenom. 144, 358-369. doi: 10.1016/S01672789(00)00087-7

Morrison, J. H., and Baxter, M. G. (2012). The ageing cortical synapse: hallmarks and implications for cognitive decline. Nat. Rev. Neurosci. 13, 240-250. doi: $10.1038 / \mathrm{nrn} 3200$

Mortimer, J. A., Snowdon, D. A., and Markesbery, W. R. (2003). Head circumference, education and risk of dementia: findings from the Nun Study. J. Clin. Exp. Neuropsychol. 25, 671-679. doi: 10.1076/jcen.25.5.671.14584

Niso, G., Bruña, R., Pereda, E., Gutiérrez, R., Bajo, R., Maestú, F., et al. (2013). HERMES: towards an integrated toolbox to characterize functional and effective brain connectivity. Neuroinformatics 11, 405-434. doi: 10.1007/s12021-0139186-1

Nolte, G., Bai, O., Wheaton, L., Mari, Z., Vorbach, S., and Hallett, M. (2004). Identifying true brain interaction from EEG data using the imaginary part of coherency. Clin. Neurophysiol. 115, 2292-2307. doi: 10.1016/j.clinph.2004.04.029

Oldfield, R. C. (1971). The assessment and analysis of handedness: the Edinburgh inventory. Neuropsychologia 9, 97-113. doi: 10.1016/0028-3932(71)90067-4

Palva, J. M., Monto, S., Kulashekhar, S., and Palva, S. (2010). Neuronal synchrony reveals working memory networks and predicts individual memory capacity. Proc. Natl. Acad. Sci. U. S. A. 107, 7580-7585. doi: 10.1073/pnas.0913113107

Palva, S., and Palva, J. M. (2007). New vistas for alpha-frequency band oscillations. Trends Neurosci. 30, 150-158. doi: 10.1016/j.tins.2007.02.001

Palva, S., and Palva, J. M. (2011). Functional roles of alpha-band phase synchronization in local and large-scale cortical networks. Front. Psychol. 2:204. doi: 10.3389/fpsyg.2011.00204

Pfeffer, R. I., Kurosaki, T. T., Harrah, C. H., Chance, J. M., and Filos, S. (1982). Measurement of functional activities in older adults in the community. J. Gerontol. 37, 323-329. doi: 10.1093/geronj/37.3.323

Raghavachari, S., Kahana, M. J., Rizzuto, D. S., Caplan, J. B., Kirschen, M. P., Bourgeois, B., et al. (2001). Gating of human theta oscillations by a working memory task. J. Neurosci. 21, 3175-3183.

Reuter-Lorenz, P. A. (2002). New visions of the aging mind and and brain. Trends Cogn. Sci. 6, 394. doi: 10.1016/S1364-6613(02)01957-5

Scarmeas, N., Zarahn, E., Anderson, K. E., Hilton, J., Flynn, J., Van Heertum, R. L., et al. (2003). Cognitive reserve modulates functional brain responses during memory tasks: a PET study in healthy yung and elderly subjects. Neuroimage 19, 1215-1227. doi: 10.1016/S1053-8119(03)00074-0

Scarmeas, N., Zarahn, E., Anderson, K. E., Honig, L. S., Park, A., Hilton, J., et al. (2004). Cognitive reserve-mediated modulation of positron emission tomographic activations during memory tasks in Alzheimer disease. Arch. Neurol. 61, 73-78. doi: 10.1001/archneur.61.1.73

Siegel, M., Donner, T. H., and Engel, A. K. (2012). Spectral fingerprints of large-scale neuronal interactions. Nat. Rev. Neurosci. 13, 121-134. doi: $10.1038 / \mathrm{nrn} 3137$

Singer, W. (1999). Neuronal synchrony: a versatile code for the definition of relations? Most of our knowledge about the functional organization. Neuron 24, 49-65. doi: 10.1016/S0896-6273(00)80821-1 
Solé-Padullés, C., Bartrés-Faz, D., Junqué, C., Vendrell, P., Rami, L., Clemente, I. C., et al. (2009). Brain structure and function related to cognitive reserve variables in normal aging, mild cognitive impairment and Alzheimer's disease. Neurobiol. Aging 30, 1114-1124. doi: 10.1016/j.neurobiolaging.2007.10.008

Sporns, O., Chialvo, D. R., Kaiser, M., and Hilgetag, C. C. (2004). Organization, development and function of complex brain networks. Trends Cogn. Sci. 8, 418-425. doi: 10.1016/j.tics.2004.07.008

Stam, C. J., Nolte, G., and Daffertshofer, A. (2007). Phase lag index: assessment of functional connectivity from multi channel EEG and MEG with diminished bias from common sources. Hum. Brain Mapp. 28, 1178-1193. doi: 10.1002/hbm.20346

Stam, C. J., and van Straaten, E. C. (2012). The organization of physiological brain networks. Clin. Neurophysiol. 123, 1067-1087. doi: 10.1016/j.clinph.2012.01.011

Steffener, J., and Stern, Y. (2012). Exploring the neural basis of cognitive reserve in aging. Biochim. Biophys. Acta 1822, 467-473. doi: 10.1016/j.bbadis.2011.09.012

Stern, Y. (2002). What is cognitive reserve? Theory and research application of the reserve concept. J. Int. Neuropsychol. Soc. 8, 448-460. doi: $10.1017 /$ S1355617702813248

Stern, Y. (2006). Cognitive reserve and Alzheimer disease. Alzheimer Dis. Assoc. Disord. 20, S69-S74. doi: 10.1097/00002093-200607001-00010

Stern, Y. (2009). Cognitive reserve. Neuropsychologia 47, 2015-2028. doi: 10.1016/j.neuropsychologia.2009.03.004

Stern, Y., Gurland, B., Tatemichi, T. K., Tang, M. X., Wilder, D., and Mayeux, R. (1994). Influence of education and occupation on the incidence of Alzheimer's disease. JAMA 271, 1004-1010. doi: 10.1001/jama.1994.03510370056032

Stern, Y., Habeck, C., Moeller, J., Scarmeas, N., Anderson, K. E., Hilton, H. J., et al. (2005). Brain networks associated with cognitive reserve in healthy young and old adults. Cereb. Cortex 15, 394-402. doi: 10.1093/cercor/bhh142

Stern, Y., Rakitin, B. C., Habeck, C., Gazes, Y., Steffener, J., Kumar, A., et al. (2012). Task difficulty modulates young-old differences in network expression. Brain Res. 1435, 130-145. doi: 10.1016/j.brainres.2011.11.061
Van Strien, J. W., Hagenbeek, R. E., Stam, C. J., Rombouts, S. A. R. B., and Barkhof, F. (2005). Changes in brain electrical activity during extended continuous word recognition. Neuroimage 26, 952-959. doi: 10.1016/j.neuroimage.2005. 03.003

Varela, F., Lachaux, J., Rodriguez, E., and Martinerie, J. (2001). The brainweb: Phase synchronization and large-scale integration. Nat. Rev. Neurosci. 2, 229-239. doi: $10.1038 / 35067550$

Wechsler, D. (1987). WMS-R: Wechsler Memory Scale-Revised: Manual. San Antonio, TX: Psychological Corporation.

Zarahn, E., Rakitin, B., Abela, D., Flynn, J., and Stern, Y. (2007). Agerelated changes in brain activation during a delayed item recognition task. Neurobiol. Aging 28, 784-798. doi: 10.1016/j.neurobiolaging.2006. 03.002

Conflict of Interest Statement: The authors declare that the research was conducted in the absence of any commercial or financial relationships that could be construed as a potential conflict of interest.

Received: 17 January 2014; accepted: 28 May 2014; published online: 13 June 2014. Citation: López ME, Aurtenetxe S, Pereda E, Cuesta P, Castellanos NP, Bruña R, Niso $G$, Maestú $F$ and Bajo $R$ (2014) Cognitive reserve is associated with the functional organization of the brain in healthy aging: a MEG study. Front. Aging Neurosci. 6:125. doi: 10.3389/fnagi.2014.00125

This article was submitted to the journal Frontiers in Aging Neuroscience.

Copyright (c) 2014 López, Aurtenetxe, Pereda, Cuesta, Castellanos, Bruña, Niso, Maestú and Bajo. This is an open-access article distributed under the terms of the Creative Commons Attribution License (CC BY). The use, distribution or reproduction in other forums is permitted, provided the original author(s) or licensor are credited and that the original publication in this journal is cited, in accordance with accepted academic practice. No use, distribution or reproduction is permitted which does not comply with these terms. 